\title{
The Origin of Adaptation in the Auditory Pathway of Locusts Is Specific to Cell Type and Function
}

\author{
K. Jannis Hildebrandt, ${ }^{1}$ Jan Benda, ${ }^{2}$ and R. Matthias Hennig ${ }^{1}$ \\ ${ }^{1}$ Behavioural Physiology and ${ }^{2}$ Theoretical Biology, Biology Department, Humboldt-Universität zu Berlin, 10115 Berlin, Germany
}

We investigated the origin of spike frequency adaptation within a layered sensory network: the auditory pathway of locusts. Spike frequency adaptation as observed in an individual neuron may arise because of intrinsic or presynaptic adaptation mechanisms. To separate the contribution of different mechanisms, we recorded from the same cell during acoustic and intracellular current stimulation. We studied three identified neuron types that are representative for each network layer and participate in processing auditory patterns and localizing sound sources. By comparing current and acoustic stimulation, three distinct patterns of the distribution of adaptation mechanisms within the sensory network emerged: (1) balanced influence of both intrinsic and presynaptic adaptation mechanisms in an interneuron that summates over several receptor afferents (TN1), (2) predominantly inhibiting input as the source for spike frequency adaptation in a cell that transmits both pattern representation and directional information (BSN1), (3) primarily intrinsic, spiketriggered adaptation currents within an interneuron coding exclusively for direction (AN2). The time courses of spike frequency adaptation differed significantly between the cells types. Using the adaptation time constants, we were able to predict signal transmission properties for the different cells. We conclude that the adaptation mechanisms differ greatly among interneurons within this sensory pathway and are a function of their role in information processing.

Key words: spike frequency adaptation; auditory system; invertebrate; signal transmission; adaptation mechanisms; temporal

\section{Introduction}

Spike frequency adaptation (SFA), the decrease in neuronal response strength during prolonged stimulation, is a feature of virtually all sensory systems. SFA plays a role in adjusting the neuron's dynamic range to the stimulus range (Brenner et al., 2000; Dean et al., 2005; Maravall et al., 2007), optimizing energy consumption (Heitwerth et al., 2005; Niven et al., 2007), and processing of time varying signals (French et al., 2001; Benda et al., 2005). The observed SFA can result from different mechanisms and sources of adaptation, such as the transduction process in receptors cells (Hudspeth et al., 2000; Gollisch and Herz, 2004; Albert et al., 2007), spike-dependent adaptation currents (Brown and Adams, 1980; Madison and Nicoll, 1984; Fleidervish et al., 1996), synaptic depression (Abbott et al., 1997; Chance et al., 1998) and inhibitory inputs (Finlayson and Adam, 1997; Ingham and McAlpine, 2005).

The net SFA observed in the intact sensory pathway will typically result from the sum of many processes occurring between the stimulus and the recording site, each of which transforms the

\footnotetext{
Received 0ct. 6, 2008; revised Jan. 8, 2009; accepted Jan. 15, 2009.

This work was supported by the German Research Foundation through SFB 618 “Theoretical Biology." We thank Dr. Martin Stemmler for critical reading of this manuscript.

Correspondence should be addressed to K. Jannis Hildebrandt, Behavioural Physiology, Biology Department, Humboldt-Universität zu Berlin, Invalidenstrasse 43, 10115 Berlin, Germany. E-mail: jannis.hildebrandt@biologie. hu-berlin.de.

J. Benda's present address: Biocenter, Department Biology II, Ludwig-Maximilian-Universität München, Großhaderner Strasse 2, 82152 Planegg-Martinsried, Germany.

DOI:10.1523/JNEUROSCI.4800-08.2009

Copyright $\odot 2009$ Society for Neuroscience $\quad$ 0270-6474/09/292626-11\$15.00/0
}

representation of sensory information, reflecting a general design principle (Laughlin, 1989; Baccus and Meister, 2004).

Disentangling the respective contributions to SFA within a pathway is a difficult task, however. So far, SFA has mainly been studied either by using sensory stimulation in vivo or current stimuli in vitro. In the latter case, intrinsic properties of isolated cells or small circuits are isolated, whereas sensory stimulation invokes adaptation mechanisms within the entire pathway. Here we present data from an insect system, the auditory pathway of locusts, in which we investigate SFA in vivo in response to both acoustic stimuli and injected currents in the same cell. We surveyed specific identified auditory interneurons in the metathoracic ganglion of the locust and identified different characteristic patterns of adaptation mechanisms for different cell types.

The auditory pathway of locusts at the level of the metathoracic ganglion is a hierarchical network with three layers: receptors and receptor-like interneurons, local neurons, and ascending neurons. We selected three exemplary interneurons from each processing level: TN1 receives monosynaptic input from receptor cells and exhibits spiking responses that are similar to those of the receptors, BSN1 is a local interneuron that represents an intermediate processing level, and AN2 is an ascending interneuron that transmits information directly to the insect's brain (Stumpner and Ronacher, 1991). On present evidence there is no direct connection between the three cell types presented here. Overall connectivity in the network appears to be low (Vogel and Ronacher, 2007), consisting of feedforward connections between the layers but no connections between neurons of the same layer (Marquart, 1985; Boyan, 1992, 1999; Vogel and Ronacher 2007). In the present study, we systematically quantified the magni- 
tude and time course of SFA for the three cell types in response to the two stimulation modes. We explore the distribution of adaptation mechanisms along the auditory pathway, discuss its possible consequences for sensory processing, and ask whether SFA predicts differences in coding of temporal aspects of signals for those cell types.

\section{Materials and Methods Preparation}

Experiments were performed on 18 adult locusts (Locusta migratoria), obtained from a commercial supplier. Legs, wings, head, the anterior part of the abdomen and the gut were removed. The animals were fixated by wax on a platform, dorsal side up. The thorax was opened dorsally to expose the metathoracic ganglion. The ganglion was put on a small $\mathrm{NiCr}$ "spoon" to minimize movements and the torso was filled with locust Ringer solution (Pearson and Robertson, 1981). All experiments were performed at room temperature $\left(22 \pm 2^{\circ} \mathrm{C}\right)$.

\section{Electrophysiology}

Intracellular recordings were obtained with sharp electrodes pulled on a horizontal puller (P-2000, Sutter Instruments) using borosilicate glass (GCF100F-10, Harvard Apparatus). Electrode tips were filled with a $3-5 \%$ solution of Lucifer yellow (Sigma-Aldrich) in $0.5 \mathrm{M} \mathrm{LiCl}$. Electrodes had resistances of $40-60 \mathrm{M}$, allowing for the large currents necessary in our experiments to pass through. After completion of experiments, the thoracic ganglia were removed, fixed in $4 \%$ paraformaldehyde, dehydrated and cleared in methylsalicylate. The stained cells were identified under a fluorescent microscope according to their characteristic morphology (Römer and Marquart, 1984; Stumpner and Ronacher, 1991). Recordings presented here were obtained from 18 cells of three distinct cell types (TN1, $n=9$; BSN1, $n=5$; AN2, $n=4$ ).

\section{Stimulation}

Each recorded cell was stimulated both acoustically and intracellularly by current injection. For both stimulation modes the range and resolution of intensity values for all experiments was chosen such that the entire response curve was appropriately covered. This was achieved by onlinespike detection and calculation of response curves. Recordings were sampled to the hard disk of a personal computer via a $20 \mathrm{kHz}$ A/D-converter (PCI-6014, National Instruments). For the input-response curves, we used pulses of $500 \mathrm{~ms}$ duration with a $2 \mathrm{~ms}$ ramp at beginning and end. Stimulation at each intensity level was repeated 10 times. To characterize coding properties of the cells under acoustic stimulation, we used a randomly amplitude modulated (RAM) stimulus of $5 \mathrm{~s}$ duration, repeated 10 times. The amplitude modulation was obtained by low-pass filtering Gaussian noise at $100 \mathrm{~Hz}$ and multiplication with a constant carrier of the same frequency as the pulse stimuli used for characterization of SFA. The SD of the RAM stimulus was in all cases $6 \mathrm{~dB}$, independent of the mean level, and the highest peak in the amplitude distribution was $18 \mathrm{~dB}$ (three times the SD) above mean.

Acoustic stimuli. Acoustic stimuli were delivered in a cage lined with sound absorbing foam via one of two speakers (D-28/2, Dynaudio) located at $90^{\circ}$ and $35 \mathrm{~cm}$ away from the preparation. Signal envelopes were digitally stored and delivered by custom-made software (LabView, National Instruments) via a $10-\mathrm{kHz}$ D/A-converter (PCI-6014, National Instruments). Subsequently, the envelopes were multiplied (multiplier: Heinecke) with a sine wave (FG-506, Voltcraft), attenuated under digital control (PA5, Tucker-Davis Technologies), and amplified (GTA 2100B, Blaupunkt). Sound intensities were calibrated with a 1.5 inch microphone (type 2209, Brüel \& Kjær). The carrier frequency for each cell was set to either 5 or $12 \mathrm{kHz}$, chosen on a cell-to-cell basis, depending on which frequency the cell was most sensitive to.

Current stimuli. Current stimuli were digitally stored and delivered by custom made software (LabView) via the recording electrode. The amplifier (SEC-05LX, npi electronic) was set to either bridge or discontinuous current clamp modus. Discontinuous switching rates were adjusted on a cell-to-cell basis to values of $15-20 \mathrm{kHz}$. In several cells both recording modes were applied to verify that the outcome of our analysis did not depend on the recording procedure.

\section{Data analysis}

From the digitized recordings, the spike times were extracted by a peak detection algorithm (Todd and Andrews, 1999).

Spike frequency estimation and input-response curves. Spike frequency $f(t)$ was estimated by taking the inverse of the interspike interval (ISI) between the preceding and the following spike at each time $t$ for every trial separately. At the time of a spike, we took the value of the preceding ISI. For all times before the first and after the last spike we set $f(t)$ to zero. The mean spike frequency $\bar{f}(t)$ and SD were obtained by averaging across trials. The onset response $f_{\text {on }}(I)$ was obtained by taking the maximum of $\bar{f}(t)$ during the first $30 \mathrm{~ms}$ after stimulus onset. The steady state response $\bar{f}_{\mathrm{ss}}(I)$ was calculated as the mean of the $30 \mathrm{~ms}$ interval starting at $420 \mathrm{~ms}$ after stimulus onset.

Characterization of spike frequency adaptation. The comparison of SFA under the two stimulation modes is complicated by the fact that SFA depends on signal intensity. For this reason, we linearly interpolated the onset and steady state input response curves to obtain values for $\bar{f}_{\text {ss }}$ at a given reference onset response $f_{\text {on }}^{\text {ref }}$ of 70 and $140 \mathrm{~Hz}$, respectively. For this purpose, we took the first value of $\bar{f}_{\text {on }}(I)$ that exceeded $f_{\text {on }}^{\text {ref }}$ and the value at the next lower intensity. By linear interpolation we obtain $I_{\text {ref }}$, for which $f_{\mathrm{ss}}\left(I_{\text {ref }}\right)$ can be calculated by linear interpolation. The adaptation ratio $r_{\mathrm{a}}$ is then given by $r_{\mathrm{a}}\left(I_{\text {ref }}\right)=f_{\mathrm{ss}}\left(I_{\text {ref }}\right) / f_{\text {on }}^{\text {ref. Propagated errors were }}$ calculated by partial differentiation for all four spike frequencies contributing to the interpolation equation.

If adaptation mechanisms intrinsic to receptors and interneurons added up linearly, the ratio for acoustic stimuli $r_{\mathrm{a}}^{\mathrm{ac}}$ in an interneuron that is monosynaptically connected to receptors should be the product of the adaptation ratio of the interneuron $r_{\mathrm{a}}^{\mathrm{cu}}$ and that of the receptor cells. Spike frequency in receptors adapts to $\sim 50 \%$ of their onset response (Benda, 2002). Thus, in the simplest case: $r_{\mathrm{a}}^{\mathrm{ac}}=0.5 \times r_{\mathrm{a}}^{\mathrm{cu}}$. Our data were compared with this relation.

The time constant of SFA was obtained by fitting an exponentially decaying function with three free parameters (onset spike frequency $\bar{f}_{\text {on }}$, adaptation time constant $\tau_{\text {adapt }}(I)$, and steady-state spike frequency $\vec{f}_{\text {ss }}$ ) to $\bar{f}(t)$ by least squares. We verified that double exponential fits did not significantly improve the fit quality. The time course of SFA proved to be strongly dependent on input intensity. For this reason $\tau_{\text {adapt }}(I)$ was pooled and displayed as histograms. For the comparison of the time course of SFA under current and acoustic stimulation (see Fig. 3), only values of $\tau_{\text {adapt }}(I)$ were taken at which the regression coefficient between fitted and real data was $>0.8$.

Quantification of adaptation effects after stimulus offset. The decrease in spontaneous activity because of SFA was quantified by a rate coefficient:

$$
c_{i}^{r}(I)=\left\langle\frac{n_{i}^{\text {after }}-n_{i}^{\text {before }}}{n_{i}^{\text {after }}+n_{i}^{\text {before }}}\right\rangle,
$$

where $n_{i}^{\text {before }}$ and $n_{i}^{\text {after }}$ indicate the number of action potentials in a 200 ms segment before and after the stimulus in the $i$ th trial at a given intensity $I$. Only those trials for which $n_{i}^{\text {after }}+n_{i}^{\text {before }}>0$ were taken for further analysis. Angle brackets indicate averaging over all such trials. For TN1, the window had to be extended to $300 \mathrm{~ms}$ to collect a significant number of spikes for evaluation.

Hyperpolarization was quantified as the minimum of the membrane potential within $200 \mathrm{~ms}$ after stimulus offset averaged over trials. The minimum was evaluated for single trials separately and the mean of the respective trials $200 \mathrm{~ms}$ before the stimulus was subtracted before averaging.

Afterhyperpolarizations. Spike shapes were obtained by taking the median of each data point within time windows $50 \mathrm{~ms}$ before and $250 \mathrm{~ms}$ after each occurrences of a spontaneous spike. Time constants of the decay of afterhyperpolarizations were acquired by fitting a single exponential function to the time course of the median spike shape from the minimum on. The minimum of the hyperpolarization was measured relative to the peak amplitude of the spikes to compensate for variations in recording site and quality.

Characterization of coding properties. To describe coding properties, we presented RAM stimuli that elicited an onset response of $\sim 250 \pm 5 \mathrm{~Hz}$. RAM stimuli were filled with the same carrier frequency as the test pulses 
presented for the characterization of SFA. To obtain an estimate of the frequency transmission properties of the cell types, the gain functions were calculated. Spike frequency responses to RAM stimuli $500 \mathrm{~ms}$ after stimulus onset were sampled in $1 \mathrm{~ms}$ bins. Subsequently, hamming windows of $1024 \mathrm{~ms}$ duration and overlapping $50 \%$ were transformed to the frequency domain and compared with the respective window in the stimulus:

$$
g(f)=\mid \frac{\left\langle R_{\mathrm{rs}}(f)\right\rangle}{\left\langle R_{\mathrm{ss}}(f)\right\rangle},
$$

where $R_{\mathrm{rs}}(f)$ is the cross-spectral density between response and stimulus, $R_{\mathrm{ss}}(f)$ is the autospectral density of the stimulus, $\left\langle{ }^{*}\right\rangle$ indicates averaging across the $1024 \mathrm{~ms}$ windows and ${ }^{*} \mid$ is the magnitude of the complex argument.

\section{Results}

Characterization and comparison of spike frequency adaptation

In the present study, we investigated whether different interneurons exhibit characteristic SFA patterns. In a first step, we characterized SFA in three auditory interneurons at different processing levels by determining the input-response relations. To do this, we obtained the onset and steady state responses to both current and acoustic pulse stimuli at different intensity levels.

\section{Response curves}

Stimulated acoustically, TN1 showed a steep sigmoid, receptor-like response curve, saturating at higher intensity levels. This was observed for both the onset and the steady state response. Spike frequency adaptation was moderate, reducing the steady state response to $\sim 30-40 \%$ of the onset response (Fig. $1 A$, left). Under current stimulation, TN1 showed a linear inputresponse relation throughout the entire range of currents from 0 to $5 \mathrm{nA}$ (Fig. $1 \mathrm{~B}$, left). The steady state response was also linear and about half that of the onset response. For the respective stimulation modes, the general shape of onset and steady state response function was the same.

BSN1 showed pronounced SFA under acoustic stimulation, had a much lower threshold than TN1, and the onset response saturated quickly. At higher intensities, the steady state response was reduced to zero, whereas the onset response remained unchanged (Fig. $1 A$, middle). The spike raster plot at the bottom of the middle panel of Figure $1 A$ displays how spiking is suppressed entirely after a short onset response at intermediate intensity levels. Under current stimulation, BSN1 generally resembled the response patterns of TN1, and exhibited a linear input-response relationship for onset and steady state response up to relatively large currents (Fig. $1 \mathrm{~B}$, middle). BSN1 saturated at higher intensities. We were able to elicit much larger responses in BSN1 than in TN1, a fact that might be attributed to the actual site of recording and may account for these differences between the two cells.

In AN2, the slope of the onset response curve during acoustic stimulation was shallower than in either BSN1 or TN1 (Fig. 1 A, right). It fired phasically in both stimulation modes; spikes at the end of the $500 \mathrm{~ms}$ stimulus occurred only at intermediate intensities. At high intensities firing ceased entirely after the onset response (Fig. $1 A$, right panel, bottom). This pattern of AN2 was similar to BSN1 under acoustic stimulation. AN2, however, showed the same response pattern under current stimulation: for larger injected currents, spikes occurred only at the beginning of the stimulus (Fig. $1 B$, right).

\section{Magnitude of spike frequency adaptation}

To quantify and compare the magnitude of SFA, we computed the ratio between steady state and onset response at both stimulation modes. In most cases, this ratio was not constant over different intensity levels that elicited different onset responses (Fig. 2A). Starting with two onset ratios for two different frequencies, we linearly interpolated the input-response relation to estimate the ratios at 70 and $140 \mathrm{~Hz}$ onset spike frequency. This enabled us to compare the magnitude of SFA under acoustic and current stimulation directly and to relate the ratios to SFA in receptor neurons, as studied by Benda (2002).

In $\mathrm{TN} 1$, the adaptation ratio for current stimulation was between 0.5 and 0.8 at almost all intensity levels (Fig. $2 A$, lower left). During acoustic stimulation, SFA was much stronger and ratios were usually $<0.5$ (Fig. $2 A$, upper left). Direct comparison of the adaptation ratios at 70 and $140 \mathrm{~Hz}$ (Fig. $2 \mathrm{~B}, \mathrm{C}$ ) showed that the magnitude of SFA to acoustic stimuli is qualitatively in agreement 
A

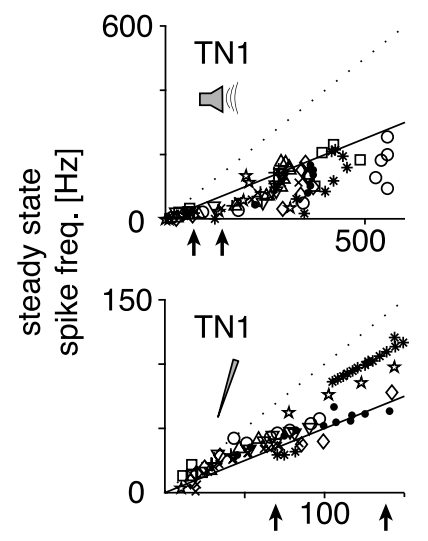

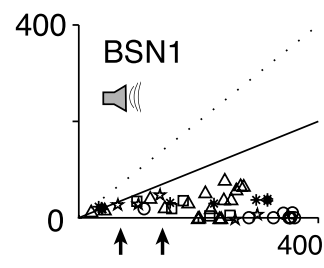

300

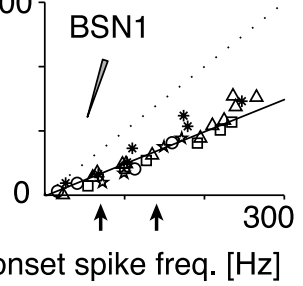

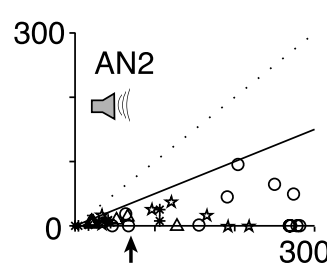

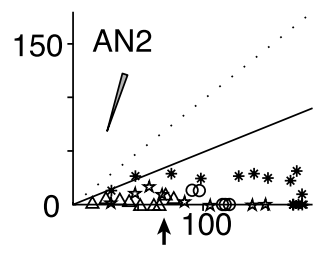

B

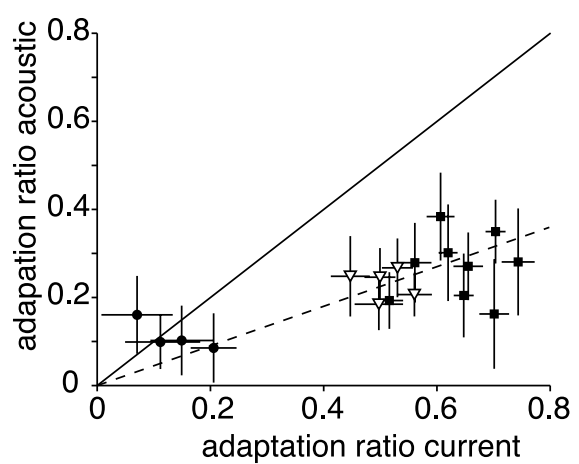

C

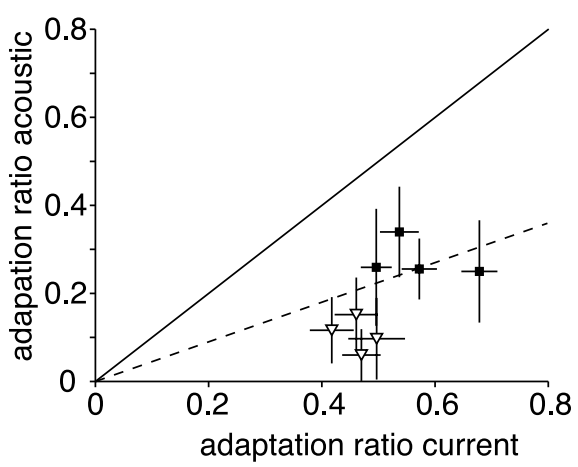

Figure 2. Magnitude of spike frequency adaptation. A, Comparison between onset and steady state spike frequency at different stimulus intensities. Different cells are represented by different symbols. In the upper (acoustic stimulation) and lower (current stimulation) graphs data from the same cells are shown. The dotted line marks a ratio $f_{s s} / f_{\max }$ of 1 , corresponding to no SFA, whereas the solid line depicts a decrease of the spike frequency by $50 \%$. The black arrows indicate those onset spike frequencies for which adaptation ratios are plotted in $\boldsymbol{B}$ and $\boldsymbol{C}(70$ and $140 \mathrm{~Hz}) . \boldsymbol{B}, \boldsymbol{C}$, Comparison of adaptation ratios $\left(f_{\mathrm{ss}} / f_{\text {on }}\right)$ for current and acoustic stimulation at a single onset spike frequency. Onset and steady state spike frequencies were derived from linear interpolations; error bars depict SD as calculated by propagation of error (see Materials and Methods). The solid line marks equal SFA under current and acoustic stimulation, the dashed line depicts the expectation from a simple linear multiplication of adaptation mechanisms in the receptors and spiking-triggered adaptation currents in the interneuron. Onset frequency $70 \mathrm{~Hz}(\boldsymbol{B})$ or $140 \mathrm{~Hz}$ (C); only those cells are shown for which in both stimulation modes a spike frequency of at least $140 \mathrm{~Hz}$ were reached. Filled circles, AN2; open triangles, BSN1; filled squares, TN1.

with a linear combination of SFA in receptors and adaptation mechanisms within TN1, leading to a multiplication of adaptation ratios. Cells exhibiting stronger SFA under current stimulation should also adapt more strongly to acoustic stimuli, yet no positive correlation between the ratios for acoustic and current stimuli was found for TN1 $(r=0.09, p=0.81$ ) (Fig. $2 B, C)$. The putative correlation may have been masked by the large errors in the input-response curves that propagated to the adaptation ratios.

In BSN1, SFA to current stimulation showed a constant ratio $\sim 0.5$ (Figs. $1 \mathrm{~B}, 2 \mathrm{~A}$ ). Under acoustic stimulation, the magnitude of SFA depended on stimulus intensity, but was always lower than 0.5 (Figs. 1A, 2A). At $70 \mathrm{~Hz}$ onset response, the ratio under acoustic stimulation was about half of that under current stimulation, similar to what was observed in TN1 (Fig. 2B). At $140 \mathrm{~Hz}$ onset response, SFA during acoustic stimulation was much stronger than expected from adaptation mechanisms within receptors and BSN1 alone, suggesting additional processes underlying the SFA at higher acoustic stimulus levels.

In AN2, the magnitude of SFA was nonlinear with respect to intensity, both under current and acoustic stimulation (Figs. $1 A, B, 2 A$ ). The adaptation ratio was in both cases $\sim 0.2$ for the 70 $\mathrm{Hz}$ response. At these levels the large errors rule out an interpretation of the relation between these two. We did not analyze the AN2 at the higher onset response, because only one of the cells under study responded with $>140 \mathrm{~Hz}$ at both stimulation modes.

\section{Time course of spike frequency adaptation}

The second important characteristic of spike frequency adaptation is its time course. During acoustic stimulation, the time course of SFA elicited by current stimulation is convolved with the kinetics of all adaptation processes peripheral to the cell, whereas in the current injection it is not. Thus, only if SFA under acoustic stimulation is dominated by intrinsic mechanisms of the cell, the time course and its intensity dependence should be similar under acoustic and current stimulation.

In TN1, the decrease of spike frequency was approximately exponential over all intensity levels (Fig. $3 A, B$ ), whereas in BSN1 this was only the case for current stimulation (Fig. 3B). In BSN1 the time course of responses to current stimuli was essentially intensity invariant (Fig. 3B), whereas higher intensity levels under acoustic stimulation led to a discontinuous, phasic firing behavior (Fig. 3A). This phasic firing pattern at certain intensity levels was observed in the AN2 for both acoustic and current stimulation (Fig. $3 A, B$ ). We quantified the time course by single exponential fits where possible. For the following analysis only data were included for which a single exponential fit yielded a regression coefficient $>0.8$. In TN1, the time courses for acoustic and current stimuli were rather similar (Fig. $3 A, B$ ) and did not differ significantly ( $p=0.37$, Wilcoxon ranks sum test; acoustic stimulation: $60.0 \pm 14.9 \mathrm{~ms}$; current stimuli: $49.3 \pm 21.6 \mathrm{~ms}$ ) (Fig. $3 C)$. In BSN1 time constants differed between current and acoustic stimulation $(p<0.01)$ : current stimuli reduced spike frequency with a time constant of $49.8 \pm 14.8 \mathrm{~ms}$, similar to TN1, whereas acoustic stimuli led to shorter time constants of $18.7 \pm$ $10.9 \mathrm{~ms}$ (Fig. 3C). In AN2, SFA acted on longer time scales than in TN1 and BSN1. Both current and acoustic stimulation revealed time constants longer than $100 \mathrm{~ms}$ and were not significantly different from each other (acoustic: $143.6 \pm 38.2 \mathrm{~ms}$, current: $123.8 \pm 35.0 \mathrm{~ms}, p=0.68$ ) (Fig. $3 C$ ).

In summary, TN1 and BSN1 showed very similar adaptation characteristics when stimulated with current and revealed a moderate and linear SFA with time constants $\sim 50 \mathrm{~ms}$. When stimulated acoustically, this pattern changed dramatically in BSN1 and became much more similar to the pattern observed for the AN2 that exhibited strong SFA with a high dependence on the stimulus level. In AN2, current and acoustic stimuli led to very similar 

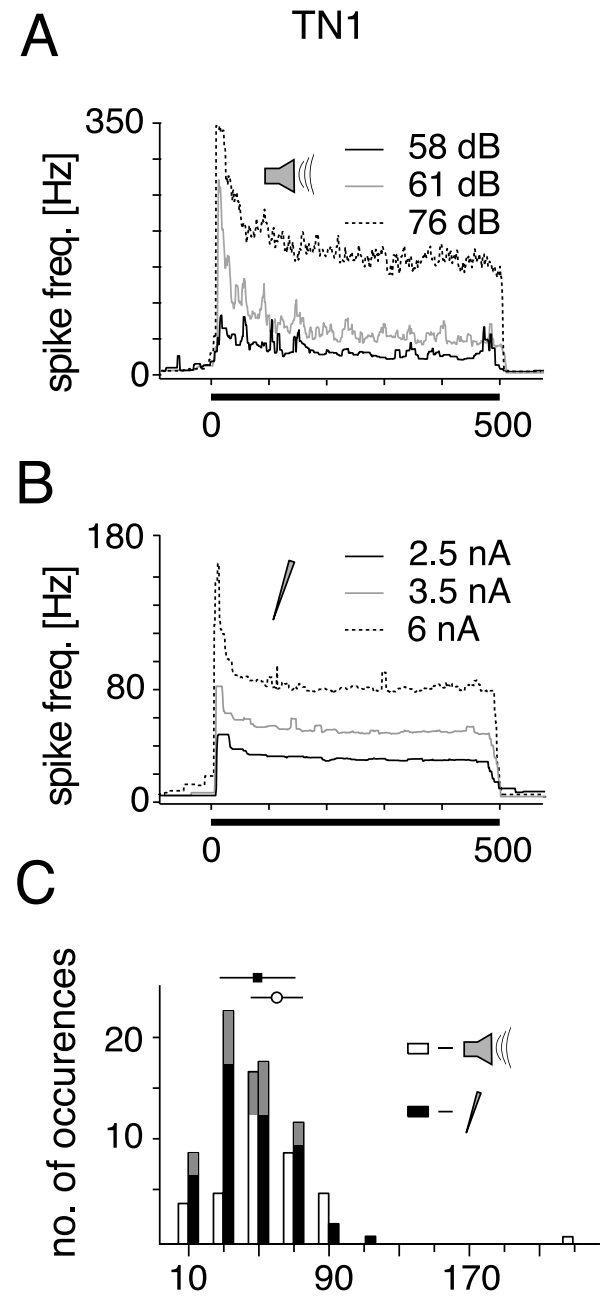

BSN1
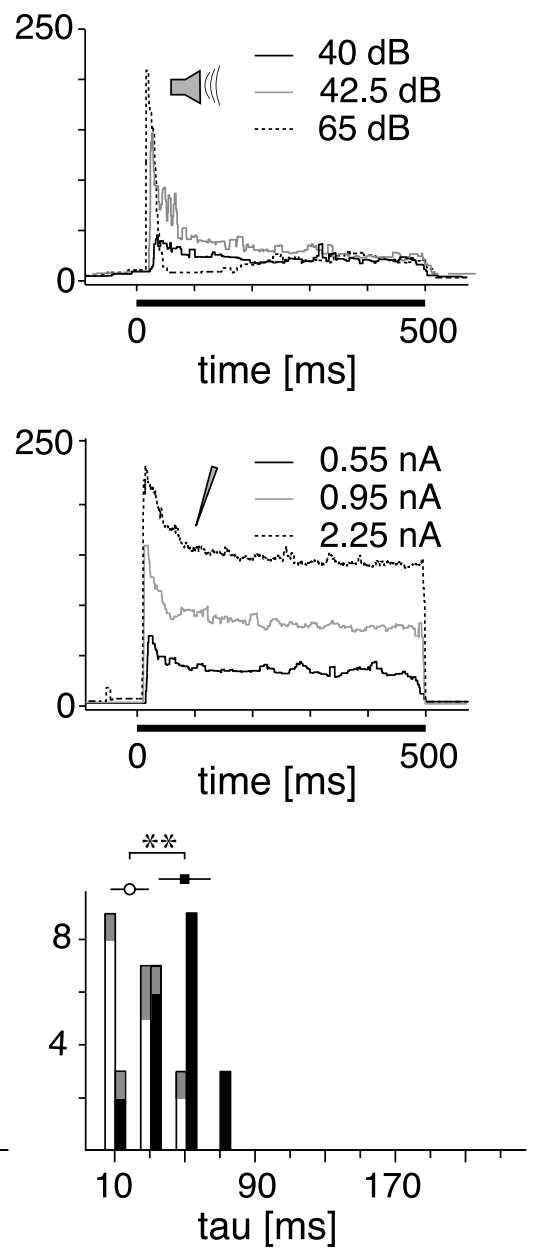

AN2
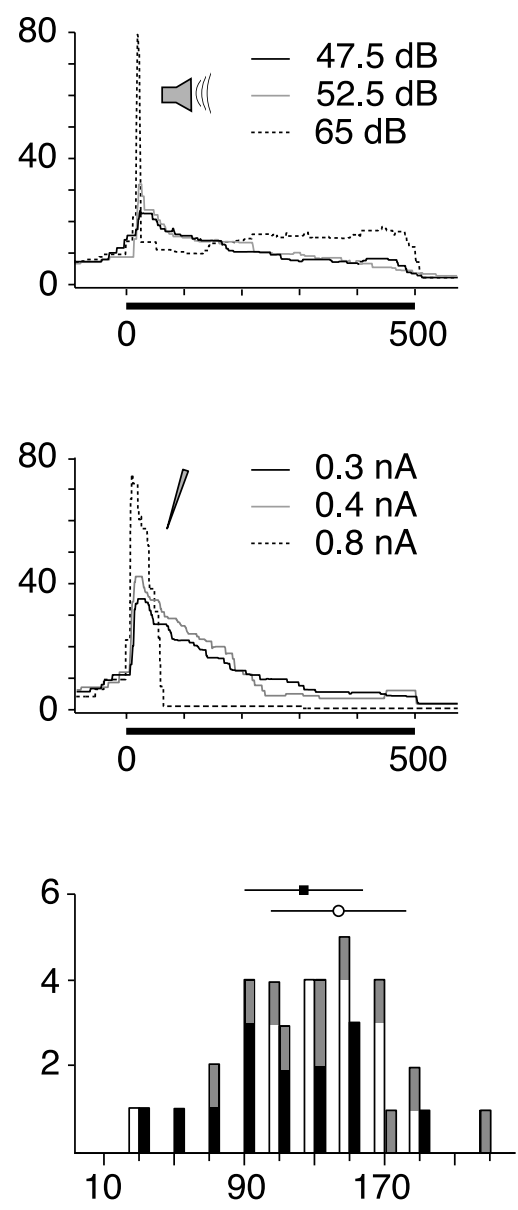

Figure 3. Time course of spike frequency adaptation during $500 \mathrm{~ms}$ constant stimulation at different intensity levels for current and acoustic stimulation. $\boldsymbol{A}, \boldsymbol{B}$, the black bar at the bottom of each graph indicates the presentation of the stimulus. The solid black line represent the lowest, the gray line an intermediate and the dashed line the highest intensity level. $\boldsymbol{C}$, Comparison of time constants of SFA. Time constants for all available stimulus presentations (data were rejected if a single exponential fit did not yield a regression coefficient $>0.8$ ). Above the histograms, means \pm SDs are indicated, asterisks mark significance at the 1\% level. All data from the cells shown in $\boldsymbol{A}$ and $\boldsymbol{B}$ are colored in gray. Open bars represent acoustic stimulation, filled bars depict current stimulation.

results with respect to time course and magnitude of SFA. These findings provide evidence for strong network effects on SFA under acoustic stimulation in BSN1, whereas in AN2 SFA can be understood mainly by intrinsic properties. In TN1, SFA observed under acoustic stimulation is in good agreement with a linear combination of adaptation mechanisms within receptors and in the interneuron itself.

\section{Hyperpolarization after stimulus offset}

Having analyzed the magnitude and time course of SFA, we aimed to reveal the mechanisms behind SFA in the cell under study, specifically hyperpolarizing currents and inhibition. If SFA affects a cell directly, the reduction in spike frequency should be observed as a decrease in excitability of a cell and should be maintained after stimulus offset. Therefore spontaneous activity and hyperpolarizations directly after the stimulus were evaluated as measures of excitability. A poststimulus hyperpolarization due to SFA can have two sources: if it is the result of intrinsic adaptation currents, then it should occur after both current and acoustic stimulation; however, only acoustic stimulation will cause an- other neuron to inhibit the observed cell, but not current injection.

The relationship between activity during stimulation and reduced excitability after stimulation as revealed by spontaneous activity was quantified by analyzing the voltage traces after stimulus offset. In TN1, the membrane potential returned to its initial value already $10 \mathrm{~ms}$ after stimulus offset for both current and acoustic stimulation (Fig. 4A). The membrane potential after acoustic stimulation displayed a very brief hyperpolarization directly after stimulus offset, which was not observed after current injection (Fig. 4A). Further inspection of the voltage traces revealed the consistent appearance of a hyperpolarization for spikes at the end of the stimulation period. These hyperpolarizations were always of the same amplitude and resembled the hyperpolarization after single spikes (see Fig. 6). These fast hyperpolarizations were not observed after current stimulation, probably because spikes seldom occurred just at the end of a stimulus due to lower firing rates in TN1 under these conditions. As a result, only a very weak or no correlation between response strength during stimulation and after stimulus hyperpolarization was observed in TN1 (Fig. 4B,E). 

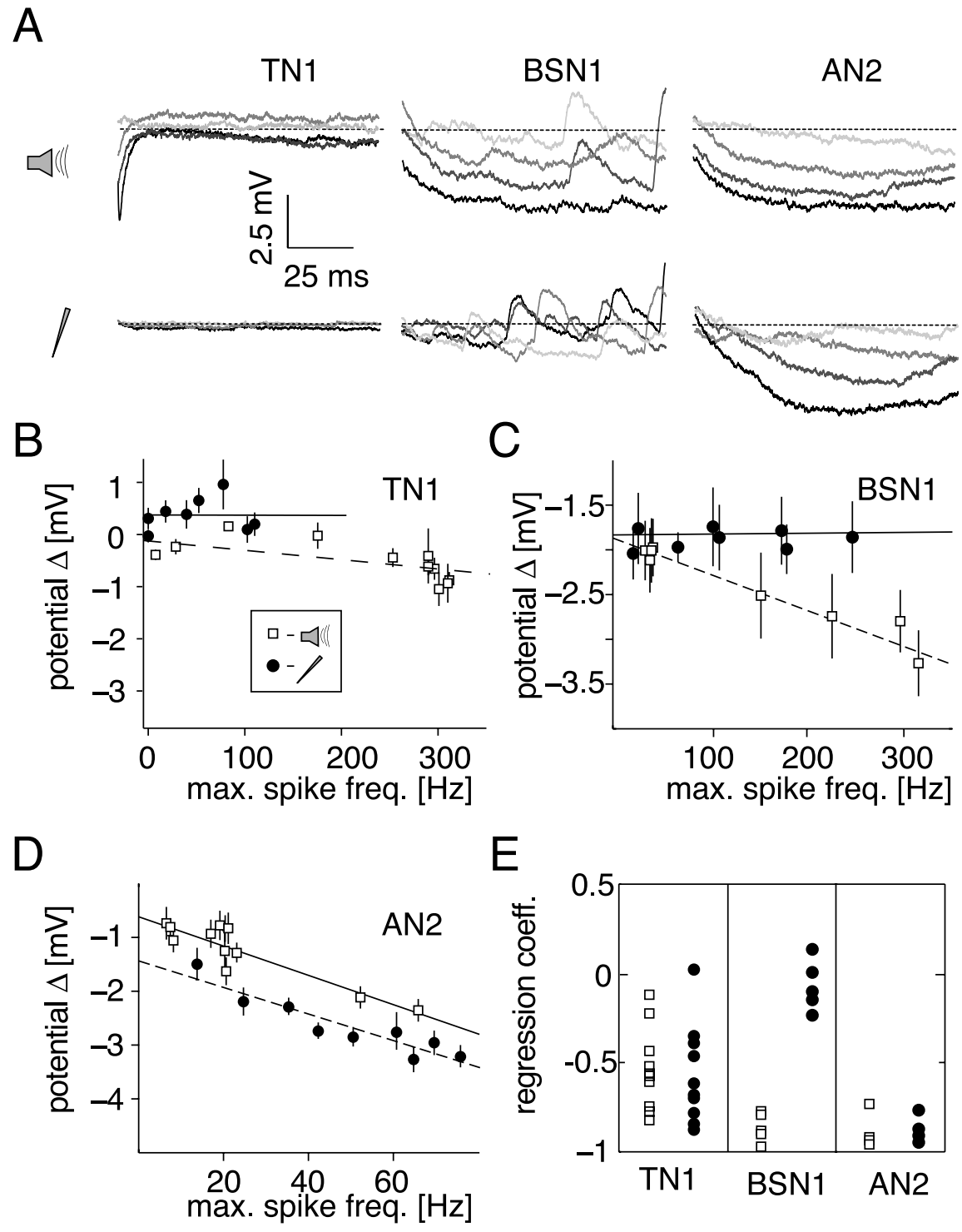

Figure 4. Hyperpolarization after stimulus offset. $\boldsymbol{A}$, Time course of the membrane potential for the first $100 \mathrm{~ms}$ after stimulus offset for different stimulus intensities. The dotted line depicts the mean potential before stimulus presentation. Darker shading represents a higher intensity of the stimulus. TN1: current, $0.5,1.0,1.5$, and $2.5 \mathrm{nA}$; acoustic, 60, 66, 72, and 78 dB. BSN1: current, $0.55,0.95,1.5$, and $2.25 \mathrm{nA}$; acoustic, 40, 47.5, 55, and $65 \mathrm{~dB}$. AN2: current, $0.5,0.6,0.7$, and $0.8 \mathrm{nA}$; acoustic 45, 55, 65, and $75 \mathrm{~dB}$. $\boldsymbol{B}-\boldsymbol{D}$, Hyperpolarization after stimulus offset as a function of the maximal spike frequency response during stimulation. Each data point depicts the mean and SD of the minimum of the membrane potential for 10 repetitions in a window 200 ms after stimulation relative to the mean potential before stimulus onset. Open symbols, Acoustic stimulation; filled symbols, current stimulation. Lines show linear fits to the data. $\boldsymbol{B}-\boldsymbol{D}$, Data from the same cells as in Fig. 5A-C). , Regression coefficients for linear fits to rate hyperpolarization versus maximal spike frequency for all recorded cells for current and acoustic stimulation.

Higher levels of acoustic stimulation led to larger hyperpolarizations in BSN1 (Fig. 4A), whereas current stimulation in BSN1 had almost no effect on the membrane potential after stimulation (Fig. 4B). This is reflected in the correlations between the response strength during and the level of the membrane potential after stimulation (Fig. 4A,C) (current: $r=0.04, p=0.92$, acoustic: $r=-0.93, p=0.0001)$. All five BSN1 cells showed this pattern (Fig. $4 E$ ). In AN2, hyperpolarization under current and acoustic stimulation increased clearly with stimulus amplitude (Fig. 4A) and correlated with response strength during stimulation (Fig. 4D) (current: $r=-0.92, p=0.00001$, acoustic: $r=$ $-0.93, p=0.0003$ ). We observed highly significant relations for all tested AN2-type cells (Fig. 4E).

Although the pronounced afterhyperpolarizations observed in AN2 provide strong evidence for spike activated adaptation currents within AN2 for both stimulation modes, the absence of long-lasting hyperpolarizations in TN1 and BSN1 after current stimulation could also be due to a site of recording distant from the spike initiating zone. TN1, for example was possibly recorded much more axonally than BSN1 and AN2 (for examples of voltage traces see supplemental Fig. 1A, available at www.jneurosci.org) and thus lasting hyperpolarizations in the dendritic part of the neuron may not have propagated into the axon. As a control, we analyzed the spontaneous activity in the three cells before and after current or acoustic stimulation. In this case, only spike numbers are evaluated, on which the actual recording site should not have an effect.

To quantify spontaneous activity, we counted spikes in windows of $300 \mathrm{~ms}$ before and after each stimulus and compared these by a rate coefficient. Negative values of this coefficient indicate a reduction of spontaneous activity due to stimulation. For the TN1 shown in Figure $5 A$ we observed no correlation between the response strength and the spontaneous activity after stimulus end during acoustic or current stimulation (acoustic: $r=-0.45$, $p=0.11$; current: $r=-0.20, p=0.38$ ). Although there was a small overall decrease in spontaneous activity after current stimulation, this decrease did not depend on spiking activity. All but one of nine cells of the type TN1 failed to exhibit a significant correlation between stimulated spiking activity and the number of spikes directly after acoustic stimulation. In seven recordings no correlation between current stimulation and subsequent spontaneous activity was observed (Fig. 5D). The time window for the TN1 was extended to $300 \mathrm{~ms}$ to collect a sufficient number of spontaneous spikes.

BSN1, however, remained less excitable and showed less spontaneous activity after the end of acoustic stimulation (Fig. 5B) $(r=-0.95, p=0.0003)$. Under current stimulation, there was no observable effect $(r=-0.39, p=0.34)$ in any of the 5 cells tested (Fig. 5D). This implied that BSN1, even though it adapted slightly under current stimulation, did not change its excitability on longer time scales; the strong effects under acoustic stimulation must have their origin earlier in the sensory pathway. AN2 remained less excitable after the end of both current and acoustic stimulation (Fig. 5D). This effect correlated strongly with the response strength during stimulation for current and acoustic stimuli (Fig. $5 C$ ) (current: $r=-0.79, p=0.002$, acoustic: $r=$ $-0.95, p=0.0001)$ and was observed in all four cells of this type (Fig. 5D). Thus, the analysis of spontaneous activity after stimulation confirmed the results obtained by quantifying afterhyperpolarizations (Fig. 4). 
We conclude that in both BSN1 and AN2, strong SFA is accompanied by a distinct hyperpolarization and reduction of excitability. For BSN1 hyperpolarization was not observed during current stimulation and is likely mediated by presynaptic sites. For AN2 hyperpolarizing currents were spike dependent and played an important role for SFA as well as the overall spiking pattern. In TN1, however, no long-lasting effect of SFA was observed.

\section{Hyperpolarization after individual spikes}

The analysis of hyperpolarization after current stimuli revealed that only the AN2 exhibits long-lasting hyperpolarizations as a result of its own spiking activity, whereas the TN1 displayed only very brief effects and the BSN1 showed almost no effect at all (Figs. 4, 5). Because afterhyperpolarization currents at the end of single spikes are thought to be one major source of intrinsic spike frequency adaptation, a close look at the spike forms can partly reveal the contribution of intrinsic adaptation currents in the cells we tested. We investigated spontaneous spikes only to minimize any extrinsic impact. The spike forms turned out to be very specific for each cell type (Fig. 6A). The TN1 exhibited a strong, but very fast decaying hyperpolarization, whereas in BSN1 only very weak hyperpo-

larizations were observed. In AN2, we found a strong hyperpolarization that also lasted much longer than in both TN1 and BSN1. The high specificity of the spike forms became apparent when population data were quantified. A fit by a single exponential function to the decay of afterhyperpolarizations showed that all three cell types differed from each other significantly at the $0.0001 \%$ significance level: $5.6 \pm 0.7 \mathrm{~ms}$ in TN1, $24.6 \pm 10.7 \mathrm{~ms}$ in BSN1 and $118.3 \pm 34.7 \mathrm{~ms}$ in AN2 (Fig. 6B). Notably for the AN2, the time constant of the decay of afterhyperpolarizations resembled that of SFA in this cell type $(123.8 \pm 35.0 \mathrm{~ms})$ (Fig. $3 C)$. The relative magnitude of afterhyperpolarizations was significantly smaller in BSN1 $(2.9 \pm 4.3 \%)$ than in both TN1 $(14.8 \pm 4.3 \%)$ and AN2 $(12.8 \pm 1.5 \%)$, whereas no significant difference between AN2 and TN1 was observed (Fig. 6). The spike forms help to understand the result of the analysis of hyperpolarization at stimulus offset: in TN1 afterhyperpolarization decays so rapidly that no effect lasting for $>10 \mathrm{~ms}$ can be observed. BSN1 displayed almost no afterhyperpolarization and thus only an extrinsic, inhibitory influence can be responsible for hyperpolarizations observed after acoustic stimulation. In AN2, slowly decaying afterhyperpolarizations during stimulation accumulate over time and lead to an activity-dependent decrease in excitability.

\section{Response characteristics under acoustic stimulation}

For this study, we chose three different cell types with different response properties, namely TN1, BSN1 and AN2. After the analysis of SFA in these neurons, we finally compared their coding properties, to determine the effect of spike frequency adaptation on signal processing. We compared the response properties by
B

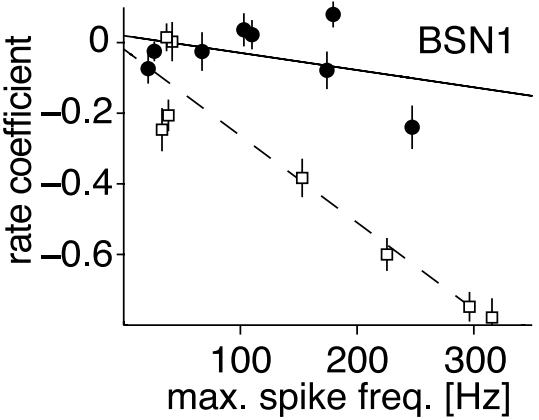

D

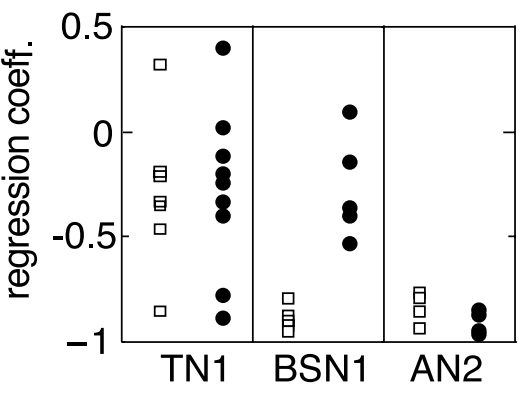


A

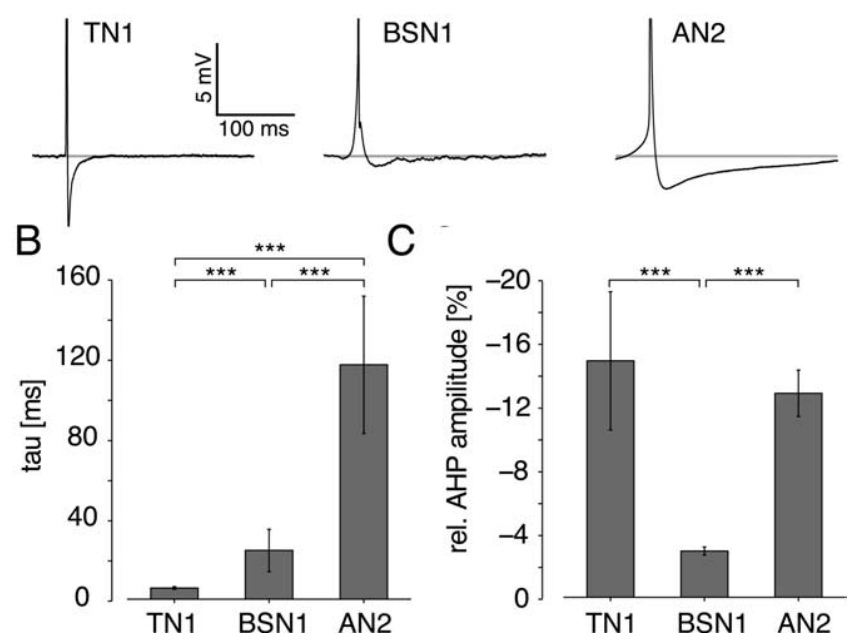

Figure 6. Spike shapes of the different cell types. $\boldsymbol{A}$, Median spike form from three exemplary cells; spikes are truncated to display afterhyperpolarization. $\boldsymbol{B}$, Mean and SD of the time constants of decay of afterhyperpolarization for all tested cells. C, Mean and SD of the minimum of afterhyperpolarization, relative to peak spike amplitude. Asterisks mark significance at the $0.1 \%$ level.

were very similar for both intensity levels, the cutoff frequency of the low-pass differed between the two recordings and depended on the mean spiking response of the cell. The high-pass characteristic, however, can be predicted by time constants of SFA. If onset and steady-state response curves were approximated by linear functions, the cutoff frequency $f^{\text {hi }}$ for transmission of AM stimuli depended only on the time constant of SFA $\tau$ and can be predicted by $f^{\text {hi }}(\tau)=(2 \pi \tau)^{-1}$ (Benda and Herz, 2003). In TN1, the gain decreased for frequencies $<3 \mathrm{~Hz}$, whereas the prediction by a mean adaptation time constant of $60.0 \mathrm{~ms}$ lied at $2.6 \mathrm{~Hz}$ (Fig. $7 B)$. For the BSN1, which adapted much more rapidly under acoustic stimulation (mean $\tau=18.7 \mathrm{~ms}$ ), we observed a higher high-pass cutoff at $10 \mathrm{~Hz}$ (predicted: $8.5 \mathrm{~Hz}$ ). The gain function of the AN2, which adapted slowly with a mean time constant of $143.6 \mathrm{~ms}$, revealed a high-pass characteristic with a cutoff between 1 and $2 \mathrm{~Hz}$ (prediction: $1.1 \mathrm{~Hz}$ ).

In summary, BSN1 and AN2 showed similar response characteristics under acoustic stimulation, shaped by a strong decrease of response strength over time. The TN1, however, responded tonically and showed stronger, but less selective locking to the time course of the stimulus.

\section{Discussion}

We examined the origin of spike frequency adaptation in different identified neurons along the auditory pathway of locusts. Between the three cell types, SFA differed significantly in strength and time course when stimulated acoustically. We were able to discern the locations of adaptation mechanisms in the sensory pathway underlying SFA by an analysis of SFA under current injection and an evaluation of changes of the membrane potential after stimulation. Thereby, we found three distinct patterns: a balanced influence of adaptation mechanisms in the afferents and intrinsic to the cells (TN1), predominately network effects via inhibition (BSN1), and primarily intrinsic, spike-triggered adaptation currents (AN2).

\section{Different origins SFA in the three cell types}

We found three different patterns on how SFA is based on mechanisms resulting from intrinsic properties or the respective pe- ripheral pathway in auditory interneurons of the locust (Fig. 8). First, both network and intrinsic cell properties can have a balanced impact on SFA as observed in TN1. In this cell, adaptation strength under acoustic stimulation was only moderately larger than under current stimulation (Fig. $2 B, C$ ). Adaptation time constants for both stimulation modes (Fig. $3 C$ ) were similar to those found in receptor cells $(10-80 \mathrm{~ms})$ (Benda, 2002) in which SFA arises mainly from encoder adaptation (Fig. 8, 2) (Benda and Herz, 2003; Gollisch and Herz, 2004). Thus, there is no evidence for an additional adaptation process other than in the afferent receptor cells and the intrinsic mechanisms of TN1 (Fig. 8, 2,4) (cf. Ocker and Hedwig, 1993).

Second, SFA can arise from network effects acting either peripherally or on the cell itself. This pattern was apparent in BSN1, which adapted quickly with time constants $<20 \mathrm{~ms}$ (Fig. $3 C$ ). The strong SFA was accompanied by long-lasting hyperpolarizations (Fig. 4A). Because these were not the result of spiking activity of the BSN1 (Fig. 4C), they probably are imposed by inhibitory inputs leading to slow hyperpolarizing currents (Fig. 8, 3) (see also Weschke and Ronacher, 2008).

Third, SFA can be dominated by intrinsic properties, as observed in AN2. This cell revealed long-lasting spike-triggered afterhyperpolarizations that accumulate over time and lead to a long-lasting reduction in excitability (Figs. 4, 6). This finding makes a spike-triggered, hyperpolarizing AHP- or M-type current the most likely mechanism behind SFA in AN2 (Fig. 8, 4) (Baldissera et al., 1973; Baldissera and Gustafsson, 1974). Notably, in AN2 SFA was stronger but slower than SFA seen in receptor neurons (Fig. 3C) (Benda, 2002). Thus, intrinsic adaptation mechanisms may overwrite the time course of SFA in the upstream pathway, provided sufficient adaptation strength.

\section{Impact of the stimulation site on the comparability between acoustic and current stimulation}

All three cells are large and show an extended dendritic tree (Römer and Marquart, 1984; Stumpner and Ronacher, 1991), and the site of current injection may have an impact on the results and their interpretation. Therefore, we compared onset responses as an indicator for the effective amount of current delivered in the two stimulation modes. In BSN1 and AN2, the maximal onset response to current and acoustic stimuli were similar (supplemental Fig. $1 B$, available at www.jneurosci.org as supplemental material), and pronounced EPSPs suggest a more dendritic position of our electrode (Fig. $4 A$; supplemental Fig. $1 A$, available at www.jneurosci.org as supplemental material).

In TN1, large sound intensities elicit very strong responses not reached by current stimulation, probably due to a more axonal position of the electrode in TN1 recordings (supplemental Fig. $1 A$, available at www.jneurosci.org as supplemental material). Thus, our conclusions for TN1 are strictly valid only for onset responses up to $150 \mathrm{~Hz}$. However, in TN1 both adaptation strength and dynamics were largely independent of intensity (Figs. 2, 3; supplemental Fig. $1 B$, available at www.jneurosci.org as supplemental material) and there is no evidence for a more complicated scheme at higher intensities [see Gabbiani and Krapp (2006) for similar results in a large visual interneuron].

\section{SFA and signal transmission properties}

Identified auditory interneurons in locusts have different temporal transmission properties (Stumpner and Ronacher, 1991; Prinz and Ronacher, 2002; Vogel and Ronacher, 2007; Wohlgemuth and Ronacher, 2007). Can SFA, which is known to be of relevance for signal recognition in grasshoppers (Ronacher and 
Hennig, 2004), be linked to known transfer characteristics of neurons?

SFA acts mainly as a high-pass filter at low frequencies (Benda et al., 2005) and the time constants of SFA predicted the observed differences in the cutoff frequency (Fig. 7C). Weschke and Ronacher (2008) also found a high-pass characteristic in BSN1 with a cutoff between 10 and $20 \mathrm{~Hz}$, which matches our findings (Fig. 7C). Notably, in AN2, the high-pass is shifted to lower frequencies than at earlier processing stages by strong but slower adaptation dynamics. This is possible, because the overall gain is lower than in more peripheral neurons (e.g., TN1).

Whereas the high-pass characteristics as reported here are difficult to compare with previous studies due to the different methods of quantification, the low-pass characteristics predicted by mean frequency confirm known cutoff frequencies (Prinz and Ronacher, 2002; Wohlgemuth and Ronacher, 2007; Weschke and Ronacher, 2008). However, to further link cell type-specific SFA to temporal processing in the respective neurons, experiments with long-duration natural stimuli are required.

\section{Functional role of the distribution of adaptation mechanisms}

Our major finding is that spike frequency adaptation acts at all levels of a sensory pathway. Similar results were reported for the locust's visual pathway (Gabbiani and Krapp, 2006) as well as for vertebrate visual (Sanchez-Vives et al., 2000a,b; Solomon et al., 2004), somatosensory (Derdikman et al., 2006, Katz et al., 2006) and auditory systems (McAlpine et al., 2000; Ingham and McAlpine, 2004, 2005; Avissar et al., 2007).

Different computational tasks may call for different distributions of adaptation mechanisms: e.g., (1) summation over many inputs to achieve high fidelity might require distributed mechanisms at several levels (Baccus and Meister, 2004; Baccus, 2006), whereas (2) converging inputs from different processing channels might require channelspecific adaptation mechanisms mostly at presynaptic stations (Abbott et al., 1997; Chance et al., 1998; Luksch et al., 2004).

In grasshoppers, the auditory pathway extracts the "what" and "where" information by processing of temporal and directional cues in parallel pathways (Hennig et al., 2004; Helversen and Helversen, 1995), and most auditory interneurons can be classified accordingly (Stumpner and Ronacher, 1994). Can the different distributions of adaptation mechanisms we present here be related to these functional aspects of auditory processing?

TN1 summates over many excitatory inputs from both sides (Stumpner and Ronacher, 1994), a process by which directional information is lost and the representation of temporal aspects enhanced. We find that mechanisms behind SFA for TN1 are distributed over several levels of processing (Fig. 8), thus matching the expectations for summation over many inputs (1) as also observed in the visual pathway of vertebrates (Solomon et al., 2004; Dunn et al., 2007).

BSN1 appears to serve both localization and pattern processing. SFA as observed in BSN1 is distinctly input dependent, as expected for the integration of several information streams in a single neuron (2). However, accumulated hyperpolarizations, probably due to inhibitory inputs, have a lasting effect. Then, SFA to a stimulus via one input affects the response to subsequent stimulation via another input and will lead to a cross-interaction of temporal and directional information.

In AN2, the intrinsic adaptation state depends mainly on the excitation of the AN2 itself and is thus largely invariant to which input triggered excitation. AN2 exhibits a strict directionality that is achieved by a balance between ipsilateral excitation and con- 

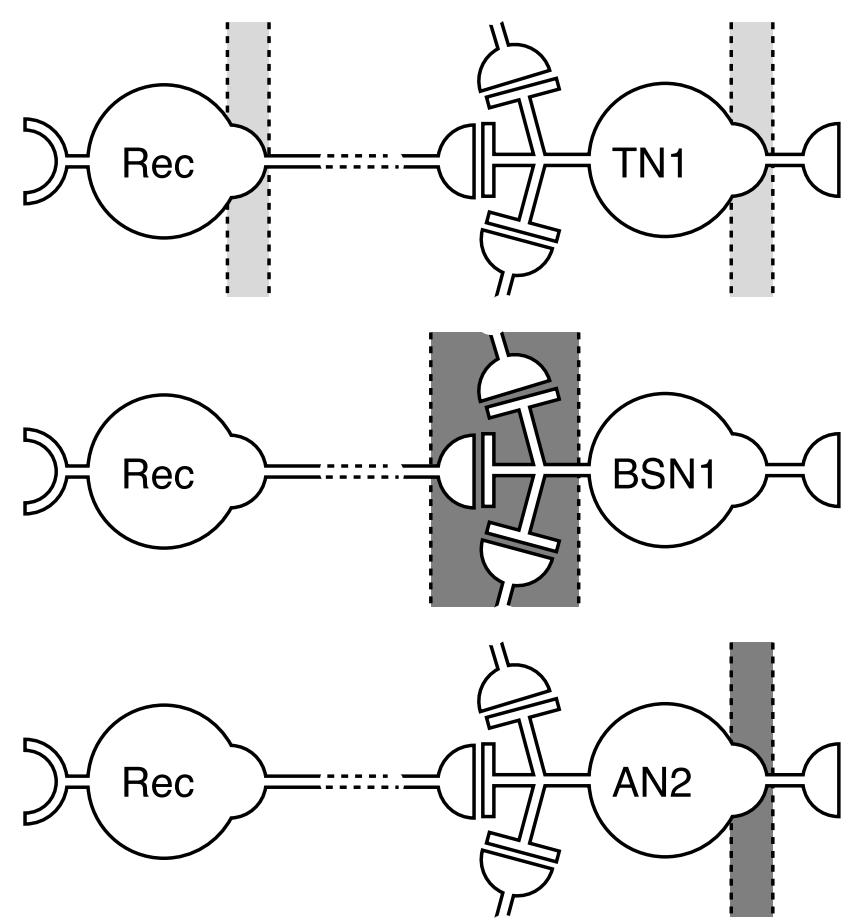

(1)

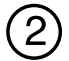

(3)

(4)

Figure 8. Distribution pattern of adaptation mechanisms within the auditory pathway. Schematic drawing of the dominating source and location of adaptation for the three cell types under study. The connection between interneurons and receptors cells can be monosynaptic but also indirect, indicated by the broken lines at their connection. The light gray areas in the sketch for TN1 represent balanced influence of two sources; dark gray areas depict one main source of adaptation. Numbers indicate mechanisms of adaptation discussed here: transducer adaptation (1), encoder adaptation in receptor neurons (2), synaptic effects (3), and encoder adaptation in the interneuron (4).

tralateral inhibition. When an adapting stimulus is presented laterally, mainly the contralateral AN2 but not the afferents to AN2 will reduce excitability, and possibly preserve the functionally important balance between inhibition and excitation. Thus, the AN2 may represent a third functional principle for the distribution of adaptation mechanisms: if the computation performed by a sensory neuron relies on the ratio of incoming inhibition and excitation, the neuron itself should adapt and not the presynaptic input.

Our analysis on the effect of SFA on coding properties revealed similar results for AN2 and BSN1 (Fig. 7); however, if directional coding is taken into account, BSN1 and AN2 display different responses (Stumpner and Ronacher, 1994) and SFA would be expected to act differently on these two neurons. Because we tested stimuli from the side that elicited a larger response for each neuron, our present investigation of SFA was restricted to temporal processing. Nevertheless, for BSN1 a lateralized adaptor could mainly decrease excitability of either contralateral inhibition or ipsilateral excitation, thereby changing the balance of these two. In AN2, excitability is mainly controlled in the postsynaptic neuron but not in its inputs and therefore directional information should be unbiased by localization of a previous stimulus.

Our results add further evidence that distributed mechanisms are a widespread feature of sensory pathways and provide an important design principle for such pathways found across taxa and modalities. Furthermore, we were able to show that the dis- tribution of adaptation mechanisms is highly specific for cell types, which in turn posses distinct coding and processing functions. Thus, the origin of SFA depends on the computational role of the part of the sensory pathway under study.

\section{References}

Abbott LF, Varela JA, Sen K, Nelson SB (1997) Synaptic depression and cortical gain control. Science 275:220-224.

Albert JT, Nadrowski B, Göpfert MC (2007) Mechanical signatures of transducer gating in the Drosophila ear. Curr Biol 17:1000-1006.

Avissar M, Furman AC, Saunders JC, Parsons TD (2007) Adaptation reduces spike-count reliability, but not spike-timing precision, of auditory nerve responses. J Neurosci 27:6461-6472.

Baccus SA (2006) From a whisper to a roar: adaptation to the mean and variance of naturalistic sounds. Neuron 51:682-684.

Baccus SA, Meister M (2004) Retina versus cortex; contrast adaptation in parallel visual pathways. Neuron 42:5-7.

Baldissera F, Gustafsson B (1974) Firing behaviour of a neurone model based on the afterhyperpolarization conductance time course and algebraical summation. adaptation and steady state firing. Acta Physiol Scand 92:27-47.

Baldissera F, Gustafsson B, Parmiggiani F (1973) Adaptation in a simple neurone model compared to that of spinal motoneurones. Brain Res 52:382-384.

Benda J (2002) Single neuron dynamics—-models linking theory and experiment. Ph.D. dissertation, Humboldt-Universität zu Berlin.

Benda J, Herz AV (2003) A universal model for spike-frequency adaptation. Neural Comput 15:2523-2564.

Benda J, Longtin A, Maler L (2005) Spike-frequency adaptation separates transient communication signals from background oscillations. J Neurosci 25:2312-2321.

Boyan GS (1992) Common synaptic drive to segmentally homologous interneurons in the locust. J Comp Neurol 321:544-554.

Boyan GS (1999) Presynaptic contributions to response shape in an auditory neuron of the grasshopper. J Comp Physiol [A] 184:279-294.

Brenner N, Bialek W, de Ruyter van Steveninck R (2000) Adaptive rescaling maximizes information transmission. Neuron 26:695-702.

Brown DA, Adams PR (1980) Muscarinic suppression of a novel voltagesensitive $K+$ current in a vertebrate neurone. Nature 283:673-676.

Chance FS, Nelson SB, Abbott LF (1998) Synaptic depression and the temporal response characteristics of V1 cells. J Neurosci 18:4785-4799.

Dean I, Harper NS, McAlpine D (2005) Neural population coding of sound level adapts to stimulus statistics. Nat Neurosci 8:1684-1689.

Derdikman D, Yu C, Haidarliu S, Bagdasarian K, Arieli A, Ahissar E (2006) Layer-specific touch-dependent facilitation and depression in the somatosensory cortex during active whisking. J Neurosci 26:9538-9547.

Dunn FA, Lankheet MJ, Rieke F (2007) Light adaptation in cone vision involves switching between receptor and post-receptor sites. Nature 449:603-606.

Finlayson PG, Adam TJ (1997) Excitatory and inhibitory response adaptation in the superior olive complex affects binaural acoustic processing Hear Res 103:1-18.

Fleidervish IA, Friedman A, Gutnick MJ (1996) Slow inactivation of Na+ current and slow cumulative spike adaptation in mouse and guinea-pig neocortical neurons in slices. J Physiol 493:83-97.

French AS, Höger U, Sekizawa S, Torkkeli PH (2001) Frequency response functions and information capacities of paired spider mechanoreceptor neurons. Biol Cybern 85:293-300.

Gabbiani F, Krapp HG (2006) Spike-frequency adaptation and intrinsic properties of an identified, looming-sensitive neuron. J Neurophysiol 96:2951-2962.

Gollisch T, Herz AVM (2004) Input-driven components of spike-frequency adaptation can be unmasked in vivo. J Neurosci 24:7435-7444.

Heitwerth J, Kern R, van Hateren JH, Egelhaaf M (2005) Motion adaptation leads to parsimonious encoding of natural optic flow by blowfly motion vision system. J Neurophysiol 94:1761-1769.

Helversen D, Helversen O (1995) Acoustic pattern recognition and orientation in orthopteran insects: parallel or serial processing. J Comp Physiol [A] 177:767-774

Hennig RM, Franz A, Stumpner A (2004) Processing of auditory information in insects. Microsc Res Tec 63:351-374.

Hudspeth AJ, Choe Y, Mehta AD, Martin P (2000) Putting ion channels to 
work: mechanoelectrical transduction, adaptation, and amplification by hair cells. Proc Natl Acad Sci U S A 97:11765-11772.

Ingham NJ, McAlpine D (2004) Spike-frequency adaptation in the inferior colliculus. J Neurophysiol 91:632-645.

Ingham NJ, McAlpine D (2005) Gabaergic inhibition controls neural gain in inferior colliculus neurons sensitive to interaural time differences. J Neurosci 25:6187-6198.

Katz Y, Heiss JE, Lampl I (2006) Cross-whisker adaptation of neurons in the rat barrel cortex. J Neurosci 26:13363-13372.

Laughlin SB (1989) The role of sensory adaptation in the retina. J Exp Biol 146:39-62.

Luksch H, Khanbabaie R, Wessel R (2004) Synaptic dynamics mediate sensitivity to motion independent of stimulus details. Nat Neurosci 7:380-388

Madison DV, Nicoll RA (1984) Control of the repetitive discharge of rat ca 1 pyramidal neurons in vitro. J Physiol 354:319-331.

Maravall M, Petersen RS, Fairhall AL, Arabzadeh E, Diamond ME (2007) Shifts in coding properties and maintenance of information transmission during adaptation in barrel cortex. PLoS Biol 5:e19.

Marquart V (1985) Neural correlations increase between consecutive processing levels in the auditory system of locusts. Naturwissenschaften 72:42-44.

McAlpine D, Jiang D, Shackleton TM, Palmer AR (2000) Responses of neurons in the inferior colliculus to dynamic interaural phase cues: evidence for a mechanism of binaural adaptation. J Neurophysiol 83:1356-1365.

Niven JE, Anderson JC, Laughlin SB (2007) Fly photoreceptors demonstrate energy-information trade-offs in neural coding. PLoS Biol 5:e116.

Ocker W, Hedwig B (1993) Serial response decrement in the auditory pathway of the locust. Zool Jahrb 97:312-326.

Pearson K, Robertson R (1981) Interneurons co-activating hindleg flexor and extensor moto-neurons in the locust. J Comp Physiol 144:391-400.

Prinz P, Ronacher B (2002) Temporal modulation transfer functions in au- ditory receptor fibres of the locust (Locusta migratoria). J Comp Physiol [A] 188:577-587.

Römer H, Marquart V (1984) Morphology and physiology of auditory interneurons in the metathoracic ganglion of the locust. J Comp Physiol [A] 155:249-262.

Ronacher B, Hennig M (2004) Neuronal adaptation improves the recognition of temporal patterns in a grasshopper. J Comp Physiol [A] 190:311-319.

Sanchez-Vives MV, Nowak LG, McCormick DA (2000a) Membrane mechanisms underlying contrast adaptation in cat area 17 in vivo. J Neurosci 20:4267-4285.

Sanchez-Vives MV, Nowak LG, McCormick DA (2000b) Cellular mechanisms of long-lasting adaptation in visual cortical neurons in vitro. J Neurosci 20:4286-4299.

Solomon SG, Peirce JW, Dhruv NT, Lennie P (2004) Profound contrast adaptation early in the visual pathway. Neuron 42:155-162.

Stumpner A, Ronacher B (1991) Auditory interneurones in the metathoracic ganglion of the grasshopper Chorthippus biguttulus: I. morphological and physiological characterization. J Exp Biol 158:391-410.

Stumpner A, Ronacher B (1994) Neurophysiological aspects of song pattern recognition and sound localization in grasshoppers. Am Zool 34:696-705.

Todd BS, Andrews DC (1999) The identification of peaks in physiological signals. Comput Biomed Res 32:322-335.

Vogel A, Ronacher B (2007) Neural correlations increase between consecutive processing levels in the auditory system of locusts. J Neurophysiol 97:3376-3385.

Weschke G, Ronacher B (2008) Influence of sound pressure level on the processing of amplitude modulations by auditory neurons of the locust. J Comp Physiol [A] 194:255-265.

Wohlgemuth S, Ronacher B (2007) Auditory discrimination of amplitude modulations based on metric distances of spike trains. J Neurophysiol 97:3082-3092. 